\title{
Ruptured heterotopic pregnancy presenting as hemorrhagic shock
}

\author{
Hannah R. Hoffman, BS ${ }^{1}$ Kristina A. Lynch, MD ${ }^{2}$ Hayden L. Smith, $\mathrm{PhD}^{1,2}$ Michael C. \\ Mintzer, MD² Sheryl M. Sahr, MD²
}

Keywords: heterotopic pregnancy, hemorrhagic shock

\begin{abstract}
Background: Heterotopic pregnancy is a rare event consisting of simultaneous intrauterine and extrauterine pregnancies. If undiagnosed, it is probable the patient will present to an Emergency Department and require emergent care.
\end{abstract}

Case Report: A 25 year-old woman arrived at the Emergency Department at 14 weeks gestation reporting sharp abdominal cramping and pelvic pain with a history of current tobacco use. Trans-abdominal ultrasound revealed a viable intrauterine pregnancy consistent with 14 weeks, closed cervix with free fluid noted in pelvis, and no evidence of ovarian torsion. Over the next three hours, the patient's condition deteriorated and hemoglobin levels dropped. The surgeon on call was consulted and suspected ruptured hepatic adenoma versus hepatic hemangioma versus visceral aneurysm. A laparotomy was emergently performed with supraceliac control of aorta to permit resuscitation, and the right and left upper quadrants of the abdominal cavity were investigated without discovery of bleeding source. Further investigation revealed ruptured left tubal ectopic pregnancy and a partial salpingectomy was performed.

Conclusions: This case serves as a clinical reminder that while heterotopic pregnancy is thought to be rare, when a patient presents with known intrauterine pregnancy and abdominal pain, heterotopic pregnancy should be included in the differential diagnosis. More common use of assisted reproductive technology may increase the incidence of heterotopic pregnancies, making familiarity with the signs, symptoms, and risk factors for this condition important.

${ }^{1}$ University of lowa Carver College of Medicine, lowa City, lowa, United States

${ }^{2}$ UnityPoint Health - Des Moines, Des Moines, lowa, United States

\section{Introduction}

A heterotopic pregnancy is a simultaneous intrauterine and extrauterine pregnancy. It is a rare event, historically occurring in 1:30,000 spontaneous conceptions. ${ }^{1}$ With the use of assisted reproductive techniques the incidence may increase to $1: 100$ to 1:500. ${ }^{2,3}$ Patients with ectopic pregnancy typically present with acuteonset abdominal pain, and imaging with ultrasound often detects the ectopic pregnancy. Transvaginal ultrasound has

Please cite this paper as: Hoffman HR, Lynch KA, Smith HL, Mintzer MC, Sahr SM. Ruptured heterotopic pregnancy presenting as hemorrhagic shock. Proc Obstet Gynecol. 2014;4(1): Article 2 [ 5 p.]. Available from: http://ir.uiowa.edu/pog/. Free full text article.

Corresponding author: Hayden L. Smith, PhD; 1415 Woodland Avenue, Suite 140, Des Moines, lowa, 50309-1453, 515241-6141, Hayden.Smith@unitypoint.org

Financial Disclosure: The authors report no conflicts of interest.

Copyright: (c) 2014 Hoffman et al. This is an open-access article distributed under the terms of the Creative Commons Attribution License, which permits unrestricted use, distribution, and reproduction in any medium, provided the original author and source are credited. 
been found to have a $99.9 \%$ sensitivity and $90.9 \%$ specificity for detecting ectopic pregnancy. ${ }^{4}$ However, an ultrasound with no evidence of ectopic pregnancy may not rule out diagnosis. The difficulty in diagnosing heterotopic pregnancy lies in the fact that the transvaginal ultrasound will provide the reassuring finding of intrauterine pregnancy, and the simultaneous ectopic pregnancy may not be seen. Providers should be suspicious of the presence of heterotopic pregnancy, especially in patients with a viable intrauterine pregnancy presenting with hemorrhagic shock.

\section{Case}

A 25 year-old G6P3023, at 14 weeks gestation arrived at the Emergency Department (ED) after experiencing acute onset of sharp abdominal cramping. Cramping continued upon admission. Patient denied any vaginal bleeding, trauma, or recent sexual intercourse. Patient was a current smoker. Patient's exam was notable for abdominal distention and tenderness without guarding. Obstetrical history for current pregnancy included a transvaginal ultrasound at 6 weeks gestation revealing a viable singleton intrauterine pregnancy (IUP) and normal adnexa. Repeat ultrasound done at 7 weeks showed normal fetal growth and no mention of adnexa. Past medical history included ovarian cysts, multiple sexual partners, multiple pregnancies, and previous dilation and curettage for an elective termination.

Trans-abdominal ultrasound revealed a viable intrauterine pregnancy consistent with gestational age of 14 weeks and a closed cervix. Free fluid was noted in the pelvis, with no evidence of ovarian torsion. Patient was treated with morphine for pain control. Within an hour and a half of arrival in the ED she complained of increasing pain, became lethargic, diaphoretic, and developed chest pain and shortness of breath. At this point, the patient's blood pressure was found to have declined from 100/52 to $63 / 30 \mathrm{mmHg}$, with heart rate ranging from 80-100 bpm. The patient's hemoglobin was $10.3 \mathrm{gm} / \mathrm{dL}$ at that time. Blood pressure responded appropriately to crystalloid resuscitation. Three hours later repeat hemoglobin returned at 7.2. Patient continued to be in severe pain, hypotensive, occasionally tachycardic, progressively lethargic, and obtunded.

Emergent surgical evaluation was obtained. Stat point-of-care hemoglobin was $4.1 \mathrm{gm} / \mathrm{dL}$. Additional vascular access was acquired and blood products were given according to the massive transfusion protocol. The patient was then taken to the operating room for an emergent laparotomy. Given the ultrasound findings of a viable IUP with the free pelvic fluid and rapid drop in hemoglobin, suspicion was raised for ruptured hepatic adenoma versus hepatic hemangioma versus visceral aneurysm. While the possibility of heterotopic pregnancy was discussed between the obstetrician and the general surgeon, it was deemed less likely than other intra-abdominal sources of hemorrhage due to the reported rarity of the condition. The patient's moribund condition precluded other studies which might have detected the heterotopic pregnancy or better defined the source of hemorrhage (i.e., computed 
tomography, magnetic resonance imaging, angiography).

An upper midline laparotomy incision was made. Massive hemoperitoneum, estimated at 3 liters, was noted upon entry into the abdomen. The right and left upper quadrants were packed. Supraceliac control of the aorta was immediately obtained to control ongoing hemorrhage and to permit the anesthesia team to catch up with resuscitation prior to continuing the exploration. The right and left upper quadrants were carefully investigated, with special attention paid to the splenic artery and the spleen, and no source of bleeding was located. The incision was then extended to the pubic symphysis and the remainder of the abdomen was also inspected. This revealed a ruptured left tubal ectopic pregnancy. A partial salpingectomy was performed. Twentynine minutes of aortic compression was required to complete the evaluation. Due to concern for the development of abdominal compartment syndrome following massive transfusion, the patient received temporary vacuumassisted abdominal closure with an ABThera $^{\mathrm{TM}}$ device. She was then admitted post-operatively to the intensive care unit and was kept intubated overnight while resuscitation continued. Patient returned to the operating room for abdominal closure the following day. She had an uneventful recovery from surgery.

An ultrasound the day after surgery showed continued viability of the IUP despite the patient's prolonged period of hemorrhagic shock and the need for aortic occlusion. A level-II ultrasound at 20 weeks was suspicious for the finding of bilateral club feet. Follow-up ultrasound at 33 weeks demonstrated poor definition of the fetal leg musculature, and subsequent ultrasound at 36 weeks showed dilation of the left ventricle of the brain. Patient underwent an elective Caesarean section at 38 weeks gestation, as she reported being anxious regarding vaginal delivery given the multiple fetal anomalies that had been identified. A liveborn female infant was delivered weighing 6 pounds 12 ounces with Apgar scores of $7 / 8$.

Subsequent neonatology evaluation showed bilateral club feet, bilateral fixed flexion of the hips and knees, and diminished muscle mass and subcutaneous tissue over the buttocks and legs. Brain magnetic resonance imaging showed diffuse cerebellar and cerebral volume loss and mild ventriculomegaly. Pediatric Neurology was consulted, and the determination was made that these sequelae were likely related to fetal hypoperfusion.

\section{Discussion}

Spontaneous heterotopic pregnancy is a very rare event, with a reported incidence of one in every 30,000 pregnancies. ${ }^{1}$ Research has shown rates of heterotopic pregnancy nearing $1 \%$ in populations using assisted reproduction techniques. ${ }^{2}$ Risk factors for ectopic pregnancy include prior ectopic pregnancy, tubal surgery, smoking more than 20 cigarettes per day, prior sexually transmitted infection, pelvic inflammatory diseases, more than 5 lifelong sexual partners, and prior medical or surgical abortion. ${ }^{5}$ The patient presented here had several risk 
factors for ectopic pregnancy, including smoking, multiple sexual partners, and prior abortion. Consideration of patient specific risk factors may be an important aspect in the diagnosis of heterotopy.

A recent article reported that, of 82 reviewed cases, $29 \%$ did not have previous risk factors associated with ectopic or heterotopic pregnancy. ${ }^{6}$ In these cases, providers frequently have lower levels of suspicion, relying more heavily on imaging to elucidate the cause of abdominal pain in pregnancy. Ultrasonography revealing an IUP plus visual evidence supportive of an ectopic pregnancy (i.e., adnexal mass, tubal ring sign), will aid in prompt diagnosis of heterotopic pregnancy. ${ }^{7}$ Conversely, inability to visualize the ectopic pregnancy may provide inappropriate reassurance to the patient and provider, delaying the diagnosis and treatment of this life-threatening condition. In the presented case, the patient had a spontaneous pregnancy and imaging revealed free fluid within the pelvis, which was consistent with an intraabdominal source for her hemorrhagic shock, but without pointing to a ruptured heterotopic pregnancy as the cause.

In ectopic pregnancy, the risk of rupture with significant hemorrhage and subsequent hemoperitoneum rises with increasing gestational age. A review of heterotopic literature published from 1994-2004 recorded that of 80 heterotopic cases, $26 \%$ were diagnosed beyond 9 weeks gestation, with $50 \%$ of the tubal sites having ruptured at the time of intervention. ${ }^{8}$ In the presented case, the patient was in her 14th week of gestation. This further complicated the diagnosis of ruptured heterotopic pregnancy.

\section{Conclusion}

This case serves as a clinical reminder that, while spontaneous heterotopic pregnancy is rare, it should be included in the differential diagnosis of a patient presenting with abdominal pain in pregnancy, especially when the patient develops signs of hemorrhagic shock. Normal hemoglobin levels can be falsely reassuring in the acutely bleeding patient, since a decrease in hemoglobin concentration requires time for equilibration to the true value. Clinical indicators such as hypotension, tachycardia, diaphoresis, and changes in mental status are far more reliable in the setting of acute hemorrhage. Early consideration of surgical exploration is strongly recommended, regardless of hemoglobin level, when these clinical indicators are observed. Given the increasing use of assisted reproductive techniques, which have a much higher incidence of heterotopic pregnancy, it is likely that ED providers will be called upon to diagnose this condition more frequently. It is therefore even more important to be familiar with risk factors, signs, and symptoms of heterotopic pregnancy. The probability may be minimal, but its repercussions can be severe.

\section{References}

1. DeVoe RW, Pratt JH. Simultaneous intrauterine and extrauterine pregnancy. Am J Obstet Gynecol. 1948 Dec;56(6):1119-26. PubMed PMID: 18893768. 
2. Tal J, Haddad S, Gordon N, TimorTritsch I. Heterotopic pregnancy after ovulation induction and assisted reproductive technologies: a literature review from 1971 to 1993. Fertil Steril. 1996 Jul;66(1):1-12. PubMed PMID: 8752602.

3. Berger MJ, Taymor ML. Simultaneous intrauterine and tubal pregnancies following ovulation induction. Am J Obstet Gynecol. 1972 Jul 15;113(6):812-3. PubMed PMID: 4635717.

4. Condous G, Okaro E, Khalid A, Lu C, Van Huffel S, Timmerman D, Bourne T. The accuracy of transvaginal ultrasonography for the diagnosis of ectopic pregnancy prior to surgery. Hum Reprod. 2005 May;20(5):1404-9. Epub 2005 Feb 3. PubMed PMID: 15695311. http://dx.doi.org/10.1093/humrep/deh77 0

5. Bouyer J, Coste J, Shojaei T, Pouly JL, Fernandez H, Gerbaud L, Job-Spira N. Risk factors for ectopic pregnancy: a comprehensive analysis based on a large case-control, population-based study in France. Am J Epidemiol. 2003 Feb 1;157(3):185-94. PubMed PMID: 12543617.

http://dx.doi.org/10.1093/aje/kwf190
6. Talbot K, Simpson R, Price N, Jackson SR. Heterotopic pregnancy. J Obstet Gynaecol. 2011;31(1):7-12. doi: 10.3109/01443615.2010.522749.

PubMed PMID: 21280985 http://dx.doi.org/10.3109/01443615.201 0.522749

7. Lin EP, Bhatt S, Dogra VS. Diagnostic clues to ectopic pregnancy. Radiographics. 2008 Oct;28(6):1661-71. doi: 10.1148/rg.286085506. PubMed PMID: 18936028.

8. Barrenetxea $G$, Barinaga-Rementeria $L$, Lopez de Larruzea A, Agirregoikoa JA,

9. Mandiola M, Carbonero K. Heterotopic pregnancy: two cases and a comparative review. Fertil Steril. 2007 Feb;87(2):417.e9-15. Epub 2006 Oct 30. PubMed PMID: 17074353. http://dx.doi.org/10.1016/j.fertnstert.200 6.05 .085 